\title{
Detecting Proteomic Indicators to Distinguish Diabetic Nephropathy from Hypertensive Nephrosclerosis by Integrating Matrix- Assisted Laser Desorption/lonization Mass Spectrometry Imaging with High-Mass Accuracy Mass Spectrometry
}

\author{
Andrew Smith ${ }^{a}$ Vadim lablokov $^{b}$ Mariafrancesca Mazza ${ }^{a}$ \\ Sonia Guarnerio ${ }^{a}$ Vanna Denti ${ }^{a}$ Mariia Ivanova ${ }^{a}$ Martina Stella ${ }^{a}$ \\ Isabella Piga ${ }^{a}$ Clizia Chinello ${ }^{a}$ Bram Heijs $^{c}$ Peter A. van Veelen ${ }^{c}$ \\ Hallgrimur Benediktsson $^{d}$ Daniel A. Muruve ${ }^{b}$ Fulvio Magni ${ }^{a}$ \\ ${ }^{a}$ Department of Medicine and Surgery, Clinical Proteomics and Metabolomics Unit, \\ University of Milano-Bicocca, Vedano al Lambro, Italy; ${ }^{b}$ Department of Medicine, \\ Cumming School of Medicine, University of Calgary, Calgary, AB, Canada; 'Center for \\ Proteomics and Metabolomics, Leiden University Medical Center, Leiden, The Netherlands; \\ ${ }^{d}$ Department of Pathology and Laboratory Medicine, Cumming School of Medicine, \\ University of Calgary, Calgary, AB, Canada
}

\section{Keywords}

Diabetic nephropathy · Hypertensive nephrosclerosis · Chronic kidney disease . Matrix-assisted laser desorption/ionization mass spectrometry imaging · Mass spectrometry · Proteomics

\begin{abstract}
Introduction: Diabetic nephropathy (DN) and hypertensive nephrosclerosis (HN) represent the most common causes of chronic kidney disease (CKD) and many patients progress to end-stage renal disease. Patients are treated primarily through the management of cardiovascular risk factors and hypertension; however patients with HN have a more favorable outcome. A noninvasive clinical approach to separate these two entities, especially in hypertensive patients who also have diabetes, would allow for targeted treatment and more appropriate resource allocation to those patients at the highest risk of CKD progression. Methods: In this preliminary study, high-spatial-resolution matrix-assisted laser desorption/ionization (MALDI) mass spectrometry imaging (MSI) was integrated with high-mass accuracy
\end{abstract}




\section{Kidney \\ Blood Pressure \\ Research}

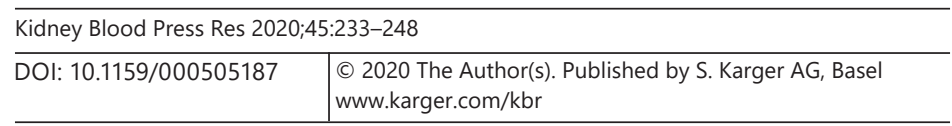

Smith et al.: MALDI-MSI to Distinguish DN from HN

MALDI-FTICR-MS and nLC-ESI-MS/MS analysis in order to detect tissue proteins within kidney biopsies to discriminate cases of DN $(n=9)$ from cases of HN $(n=9)$. Results: Differences in the tryptic peptide profiles of the 2 groups could clearly be detected, with these becoming even more evident in the more severe histological classes, even if this was not evident with routine histology. In particular, 4 putative proteins were detected and had a higher signal intensity within regions of DN tissue with extensive sclerosis or fibrosis. Among these, 2 proteins (PGRMC1 and CO3) had a signal intensity that increased at the latter stages of the disease and may be associated with progression. Discussion/Conclusion: This preliminary study represents a valuable starting point for a future study employing a larger cohort of patients to develop sensitive and specific protein biomarkers that could reliably differentiate between diabetic and hypertensive causes of CKD to allow for improved diagnosis, fewer biopsy procedures, and refined treatment approaches for clinicians.

(C) 2020 The Author(s)

Published by S. Karger AG, Basel

\section{Introduction}

Individuals with chronic kidney disease (CKD) are at risk of progression to end-stage renal disease (ESRD), which requires life-saving dialysis or renal transplantation. The most common causes of CKD in developed countries are diabetes mellitus and hypertension [1]. Diabetic kidney disease, or diabetic nephropathy (DN), accounts for approximately $44 \%$ of individuals requiring dialysis in Western countries [2]. Hyperglycemia in diabetic patients damages glomerular blood vessels, decreases the glomerular filtration rate (GFR), and increases macromolecule filtration. Injured glomeruli result in albuminuria, which is associated with a more rapid decrease in GFR and progression of CKD. Poorly controlled hypertension, on the other hand, can cause a subtype of kidney disease known as hypertensive nephrosclerosis (HN), which can also lead to ESRD. In these cases, hypertension is thought to primarily damage the arteries and arterioles of the kidney, which leads to glomerulosclerosis. Both CKD subtypes lead to ESRD over time; however, DN is associated with a yearly decline in the GFR that is roughly 4 times that of HN [3]. Clinicians may have difficulty confirming the cause of CKD in patients presenting with albuminuria who have both hypertension and diabetes, and they cannot reliably predict who will rapidly progress to ESRD. Differentiating between DN and HN has become especially important with the advent of sodium-glucose cotransporter-2 (SGLT2) inhibitors which have recently been proven to effectively reduce CKD progression in type 2 diabetics but not in patients with HN [4]. The inability to differentiate between DN and HN stems from a lack of safe, cost-effective, sensitive, and specific clinical biomarkers. Clinically, CKD is staged by estimated GFR (eGFR) and albuminuria. A lower eGFR and greater albuminuria levels are both indicative of progressive kidney dysfunction and they are synergistic [5]. While proteinuria and other clinical signs can offer clues, DN and HN are indistinguishable based on eGFR and albuminuria alone, especially in $\mathrm{HN}$ patients who have a component of secondary focal segmental glomerulosclerosis. As a result, most patients are simply treated the same or referred early to nephrologists, which may result in excessive diagnostic testing and unnecessary specialist follow-up especially for patients with nondiabetic HN and a lower risk of progression [6]. Alternatively, a kidney biopsy can be performed to confirm or refute either diagnosis, but this procedure is associated with a $6-8 \%$ risk of major bleeding complications and it is not an ideal solution [7].

Biomarkers that distinguish DN from $\mathrm{HN}$ would result in more precise patient care and better resource utilization, especially as it pertains to SGLT2 inhibition. A biomarker that can correctly differentiate between these 2 causes of CKD would lead to targeted therapy and 
Table 1. Patient characteristics of the sample cohort analyzed by MALDI-TOF- MSI

\begin{tabular}{lcc}
\hline Characteristic & DN & HN \\
\hline Age, years & $68 \pm 4.8(n=9)$ & $57 \pm 5.2(n=9)$ \\
Male gender, $\%$ & 44 & 44 \\
ACR, mg/mmol & $249.7 \pm 118.2(n=6)$ & $39.8 \pm 31.8(n=4)$ \\
Cr, $\mu \mathrm{mol} / \mathrm{L}$ & $235.7 \pm 53.8(n=9)$ & $219.6 \pm 40.94(n=8)$ \\
$\mathrm{eGFR}, \mathrm{mL} / \mathrm{min} / 1.73 \mathrm{~m}^{2}$ & $33.2 \pm 7.4(n=9)$ & $32.3 \pm 6.7(n=8)$ \\
$\mathrm{UDP}, n$ & $2(n=9)$ & $3(n=6)$ \\
UDR, $n$ & $1(n=9)$ & $1(n=4)$ \\
\hline
\end{tabular}

Values are presented as means \pm SEM unless otherwise stated. $\mathrm{Cr}$, creatinine.

focused monitoring for clinicians. Furthermore, if detectable in blood or urine, biomarkers would allow for early disease identification and intervention, thus improving health care.

The discovery of urinary biomarkers for diabetic subtypes of CKD is a growing field of clinical research and fragments of collagen type I (COL I) have been reported to be excreted more in the urine of patients with DN [8]. However, while urine samples are convenient for clinical screening, they can often be difficult to process and they can contain many proteins which are not produced by the kidney itself [9]. Matrix-assisted laser desorption/ionization (MALDI) mass spectrometry imaging (MSI) represents an ideal tool for the discovery of biomarkers that originate in renal tissues given that it combines the chemical specificity of MS with the imaging characteristics of traditional histology [10], generating thousands of ion images per single experiment and offering a molecular dimension to routine histopathology in complex renal tissue [11]. In fact, MALDI-MSI has already been shown to be capable of playing a key role in the identification of diagnostic and prognostic markers in kidney disease [12-14]. Such protein signatures can then be translated to clinically useful biomarkers present in blood or urine.

In this pilot study, we aimed to use high-spatial-resolution MALDI-TOF-MSI and, integrating it with high-mass accuracy MALDI-FTICR-MS and nLC-ESI-MS/MS analysis, to discover tissue proteins capable of differentiating DN from HN.

\section{Materials and Methods}

\section{Patient Selection}

Patients with a histological diagnosis of DN or hypertensive HN were randomly selected from the Biobank for the Molecular Classification of Kidney Disease (BMCKD) at the University of Calgary, Alberta, Canada [15]. Tissue samples in the BMCKD database represent the secondary use of specimens collected for clinical care under a protocol approved by the Conjoint Health Research Ethics Board at the University of Calgary. Samples are coded and do not contain any patient-identifying data, and thus patient consent was not required. Available clinical patient data was obtained retrospectively from Calgary Lab Services using an anonymous coding system and existing data sources. Deidentified patient data was requested at the time of biopsy and included age, sex, pathologic diagnosis, date of biopsy, eGFR, random urine albumin:creatinine ratios (ACR), urinalysis dip positivity for protein (UDP) and red blood cells (UDR). These clinical parameters were then used to characterize and compare 9 matched samples from the DN and HN group (Table 1). Samples were further classified into mild, moderate, and severe DN or HN by a renal pathologist. This was performed by scoring the biopsies on the basis of percentage 


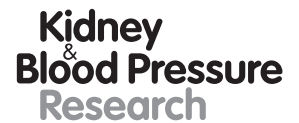

Kidney
Blood Pressure
Research

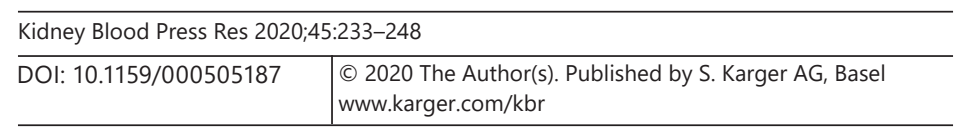

Smith et al.: MALDI-MSI to Distinguish DN from HN

interstitial fibrosis, tubular atrophy, glomerulosclerosis, and interstitial inflammation. Some clinical data was not completely available for all of the subjects, however (see below). Patient characteristics were compared using $t$ tests for continuous variables such as age, ACR, creatinine, and eGFR, and the Mann-Whitney test was used for ordinal data such as UDP and UDR.

\section{Sample Preparation for MALDI-TOF-MSI}

For this analysis, the fixation time was set at $12 \mathrm{~h}$ following the biopsy procedure, as previously described [16]. Five-micrometer-thick sections were cut and mounted onto conductive indium tin oxide glass. Paraffin removal and antigen retrieval were performed as previously described [16]. Then, trypsin deposition (20 ng/ $\mu \mathrm{L}$; Sigma-Aldrich) was performed using the iMatrixSpray (Tardo Gmbh, Subingen, Switzerland) automated spraying system and then left in a humid chamber overnight at $40^{\circ} \mathrm{C}$. Finally, matrix deposition for MALDI-MSI analysis was performed by spraying 6 layers of $\alpha$-cyano-4-hydroxycinnamic acid $(10 \mathrm{mg} / \mathrm{mL}$ in 50:50 acetonitrile:water w/0.4\% trifluoroacetic acid) using the iMatrixSpray (Tardo) with an optimized method and an incorporated heat bed set at $40^{\circ} \mathrm{C}[14]$.

\section{MALDI-TOF-MSI Analysis}

For each tissue section, mass spectra were acquired in reflectron positive mode, within the mass range of $m / z$ 700-3,000, using a rapifleX MALDI Tissuetyper ${ }^{\mathrm{TM}}$ (Bruker Daltonics GmbH, Bremen, Germany) MALDI-TOF/TOF MS equipped with a Smartbeam 3-D laser operating at a $5-\mathrm{kHz}$ frequency. A mixture of standard peptides within the mass range of $\mathrm{m} / \mathrm{z}$ 750-3,150 (PepMix I; Bruker Daltonics) was used for external calibration. MALDI-MS images were acquired with a single-spot laser setting of $18 \mu \mathrm{m}$ and a raster sampling of $20 \mu \mathrm{m}$ in both $x$ and $y$ dimensions.

\section{MALDI-TOF-MSI Data Analysis}

Data files containing the individual spectra of each entire measurement region $(115,068$ for DN and 96,539 for HN, respectively) were then imported into SCiLS Lab 2016b software (Bruker Daltonics) to perform preprocessing: baseline subtraction (convolution algorithm), normalization (total ion current algorithm), and spatial denoizing. Average (avg) spectra, representative of the whole measurement regions were generated to display differences in the protein profiles. Peak picking and alignment were performed as feature extraction for statistical analysis and this resulted in the detection of $281 \mathrm{~m} / \mathrm{z}$ features within the dataset. Unsupervised principal component analysis (PCA) was also performed to reduce the high complexity of the data. Finally, receiver operating characteristic (ROC) analysis was performed, with an area under the curve (AUC) of $\geq 0.80$ and a $p$ value $\leq 0.05$ being required for a peak to be considered as statistically significant. These signals were curated to only include $m / z$ values representative of the monoisotopic mass of a tryptic peptide.

\section{Sample Preparation for MALDI-FTICR-MS and nLC-ESI-MS/MS Analysis}

For MALDI-FTICR-MS and nLC-ESI MS/MS analysis, replicate tissue sections of all of the specimens previously analyzed by MALDI-MSI were prepared as previously described in Sample Preparation for MALDI-TOF-MSI. The matrix was removed from the tissue by washing with a solution containing 50:50 acetonitrile:water w/0.4\% trifluoroacetic acid and subsequently collected. All of the material obtained from the DN and HN specimens was pooled together, respectively. The resulting solutions were concentrated using an HETO vacuum concentrator until a final elution volume of approximately $20 \mu \mathrm{L}$ was reached. This concentrated solution was brought to a final volume of $120 \mu \mathrm{L}$ by resuspending in phase $\mathrm{A}(98 / 2 / 0.1$; water/acetonitrile/trifluoroacetic acid). The solution was then stocked at $-20^{\circ} \mathrm{C}$ prior to the MALDI-FTICR-MS and nLC-ESI-MS/MS analyses. 


\section{Kidney \\ Blood Pressure \\ Research}

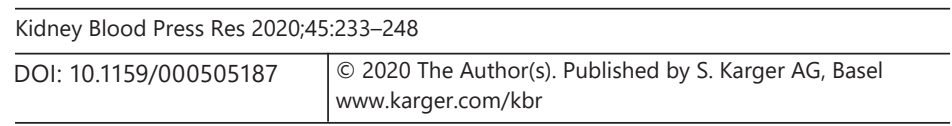

Smith et al.: MALDI-MSI to Distinguish DN from HN

\section{MALDI-FTICR MS Analysis}

A volume of $0.4 \mu \mathrm{L}$ taken from each pooled sample was spotted onto an MTP Ground Steel MALDI target plate. MALDI-FTICR-MS profiling was performed on a 12-Tesla SolariX XR mass spectrometer (Bruker Daltonics, Bremen, Germany) operating in positive-ion mode, using 150 laser shots per spot and a 100- $\mu \mathrm{m}$ laser spot size. Spectra were recorded in the $\mathrm{m} / \mathrm{z}$ range of $700-3,500$ with a $512 \mathrm{k}$ data point transient (1.1 s in duration), corresponding to an estimated resolution of 200,000 at $\mathrm{m} / \mathrm{z} 400$. Data acquisition was performed using ftmsControl (Bruker Daltonics).

\section{MALDI-FTICR MS Data Analysis}

Raw data files were uploaded into Compass DataAnalysis 4.1. Individual spectra were then exported in ASCII format, transformed into tab delimited files, and individually imported into mMass version 5.5.0 (freely available at www.mmass.org). Peak picking for each spectrum was performed by setting a relative intensity threshold (base peak) of $2.5 \%$. The peak lists were treated with a deisotoping algorithm set to remove isotope peaks with a maximal charge of $2^{+}$and an isotope mass tolerance of $0.02 \mathrm{Da}$. The resulting peak list was then used to assign an accurate mass to those $m / z$ signals previously detected by MALDI-TOF-MSI analysis.

\section{nLC-ESI-MS/MS Analysis}

Each of the tryptic peptide extracts (DN and HN) was analyzed using an Easy nLC1000 coupled to an Orbitrap Fusion ${ }^{\mathrm{TM}}$ Lumos $^{\mathrm{TM}}$ mass spectrometer. Firstly, $5 \mu \mathrm{L}$ of each sample was diluted in $15 \mu \mathrm{L}$ of $0.1 \%$ formic acid and $7 \mu \mathrm{L}$ of each fraction was injected onto an in-house-prepared precolumn ( $100 \mu \mathrm{m} \times 15 \mathrm{~mm}$; Reprosil-Pur C18-AQ $3 \mu \mathrm{m})$ and eluted via a homemade analytical column $(25 \mathrm{~cm} \times 75 \mu \mathrm{m}$; Reprosil-Pur C18-AQ $3 \mu \mathrm{m})$. The gradient went from 98 to $64 \%$ of phase A $(0.1 \%$ formic acid) over the course of $120 \mathrm{~min}$. Phase B was $0.01 \%$ formic acid:acetonitrile (80:20). The analytical column was drawn to a tip of $\sim 5 \mu \mathrm{m}$ and acted as the electrospray needle of the MS source. The Lumos mass spectrometer was operated in top n mode for $3 \mathrm{~s}$. Parameters were as follows: full scan, resolution of 120,000, AGC target of 4,000,000, and maximal fill time of $50 \mathrm{~ms}$; MS/MS, resolution of 30,000, AGC target of 500,000, and maximal fill time of $60 \mathrm{~ms}$; and intensity threshold of 25,000. The allowed charges were 1-4 and excluded after $n=1$ for $60 \mathrm{~s}$. The Thermo.RAW files were converted to MGF files using the MSconvert software (64-bit for Windows, http://proteowizard.sourceforge.net).

\section{nLC-ESI-MS/MS Data Analysis}

Proteins were identified by performing a database search using in-house Mascot software (version 2.4.1) and Swissprot database (accessed in June 2018; 557,491 sequences; $198,312,666$ residues), employing a peptide tolerance of $10 \mathrm{ppm}$ and an MS/MS tolerance of $20 \mathrm{mmu}$. Trypsin was set as the digestive enzyme, with no fixed modifications, and 1 missed cleavage was allowed. The following variable modifications were selected: methionine oxidation and 2 modifications following FFPE treatment $(+12$ and $+30 \mathrm{Da})$. An automatic decoy database search and a built-in Percolator algorithm were applied. Only peptide sequences matched with $p \leq 0.05$ were considered positive identifications. The protein identification lists for DN and HN were exported in CSV format, respectively, and data was filtered in order to obtain a list of proteins specific for each disease class.

\section{Integration of MALDI-TOF-MSI Data with nLC-ESI-MS/MS Protein Identification}

These accurate mass measurements for the MSI signals of interest were then aligned with the mass values belonging to the positively identified peptide sequences obtained using nLC-ESI-MS/MS. A protein identification was putatively assigned to a signal if an error of less 


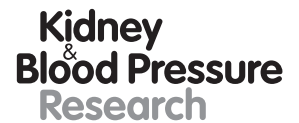

Kidney
Blood Pressure
Research

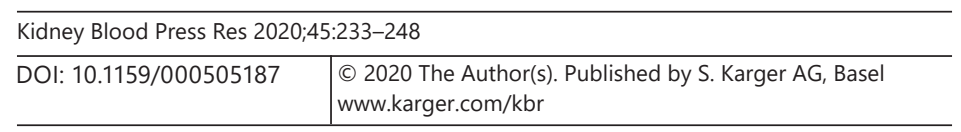

Smith et al.: MALDI-MSI to Distinguish DN from HN

than \pm 10 ppm was found between the $m / z$ value observed in MALDI-FTICR MS and the $m / z$ of the related amino acidic sequence determined by nLC-ESI-MS/MS.

\section{PGRMC1 and C3 Immunohistochemistry}

Formalin-fixed and paraffin-embedded kidney biopsies were obtained from the BMCKD. Tissue sections were cut at $7 \mu \mathrm{m}$ onto Superfrost Plus microscope slides (ThermoFisher, Waltham, WA, USA). Tissue sections were then deparaffinized and washed in distilled water. Antigen retrieval was accomplished by incubating sections in a solution containing $10 \mathrm{~mm}$ Tris base and $1 \mathrm{~mm}$ EDTA with a pH of 9.0 in a steamer for $15 \mathrm{~min}$. Slides were then allowed to cool in a heated solution for an additional $35 \mathrm{~min}$ and washed in Tris-buffered saline (TBS). Endogenous peroxidase activity was blocked with Bloxall solution (SP-6000; Vector Laboratories, Burlingame, CA, USA) according to the manufacturer's instructions. Sections were incubated with ImmPRESS blocking serum (MP7401; Vector) for $60 \mathrm{~min}$, followed by a 60-min incubation with 1:1,000 rabbit anti-human PGRMC-1 (HPA002877; Sigma-Aldrich, Oakville, ON, Canada;). Sections were then washed in TBS and incubated with ImmPRESS anti-rabbit IgG (MP-7401; Vector) following the manufacturer's instructions. Following a wash in TBS the sections were developed with ImmPACT Dab substrate for $80 \mathrm{~s}$ (SK-4105; Vector) and washed in distilled water. Finally, sections were counterstained with hematoxylin, dehydrated, and mounted with Permount. Staining for C3 was performed on frozen sections cut at $7 \mu \mathrm{m}$ using an Omnis IHC slide stainer (Dako, Mississauga, ON, USA) using the manufacturer's instructions and a prediluted FITC anti-C3 primary antibody (760-2686; Roche, Basel, Switzerland). The PGRMC1 staining intensity was quantified by the Aperio Positive Pixel Count Algorithm (Leica Biosystems, Concord, ON, Canada).

\section{Results}

The primary aim of this pilot study was to evaluate the possibility to integrate the capabilities of high-spatial-resolution MALDI-MSI with complementary, high-mass accuracy MS techniques in order to detect in situ proteomic alterations that could distinguish DN from HN.

\section{Patient Characteristics}

Nine patients with DN and 9 patients with HN were randomly selected from the BMCKD database (Table 1). Males accounted for $44 \%$ of the individuals in both groups. There were no significant differences in age, serum creatinine, eGFR, urinalysis dip positive protein, or blood. Although patients with HN generally had lower ACR, the differences were not statistically significant due to the small numbers. ACR values were not available for $3 \mathrm{DN}$ and $5 \mathrm{HN}$ subjects. UDP and UDR were not available for 3 and 5 HN subjects, respectively. The histological characteristics of this case series are presented in Figure 1 using periodic acid-Schiff (PAS) staining.

\section{Proteomic Signatures of DN and HN}

Initially, unsupervised PCA was performed on the entire MALDI-TOF-MSI dataset (DN and $\mathrm{HN}, n=18$ ) in order to highlight any proteomic differences between the 2 disease groups. As shown in Figure 2A, the majority of the spectra from the 2 disease groups were distributed in the same region of the PCA score chart (green circle) and thus can be considered to have similar proteomic profiles. However, the spectra from the DN group (red dots) displayed a more heterogeneous distribution with respect to the HN group (blue dots) and, in fact, there was a region of the PCA score chart where spectra deriving primarily from DN tissue were clustered (black circle), thus suggesting additional alterations of the proteome in this disease. 
Kidney

Blood Pressure

Research

Fig. 1. PAS-stained renal biopsies of our DN and HN case series.

\begin{tabular}{l|l}
\hline Kidney Blood Press Res 2020;45:233-248 \\
\hline DOI: 10.1159/000505187 & $\begin{array}{l}\text { ○ 2020 The Author(s). Published by S. Karger AG, Basel } \\
\text { www.karger.com/kbr }\end{array}$ \\
\hline
\end{tabular}

Smith et al.: MALDI-MSI to Distinguish DN from HN

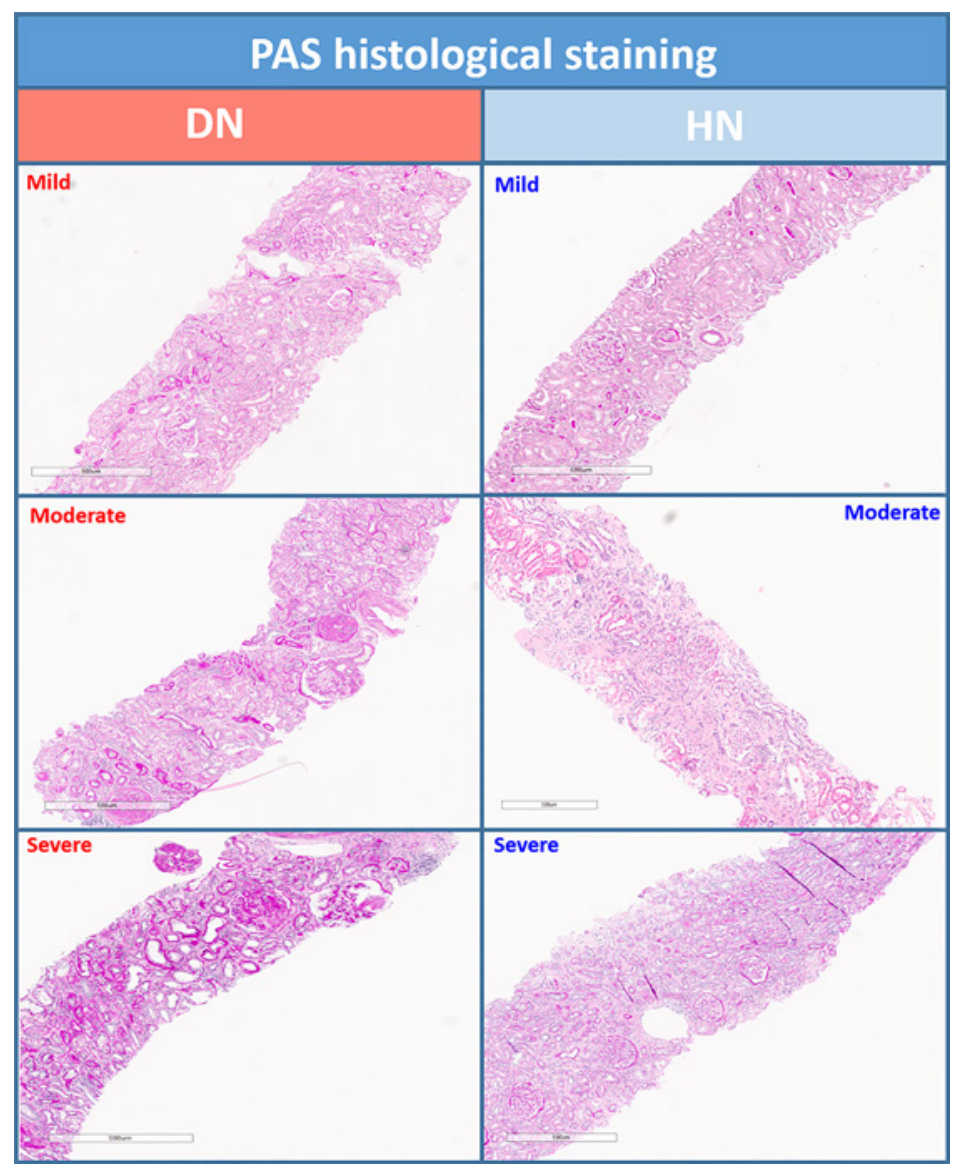

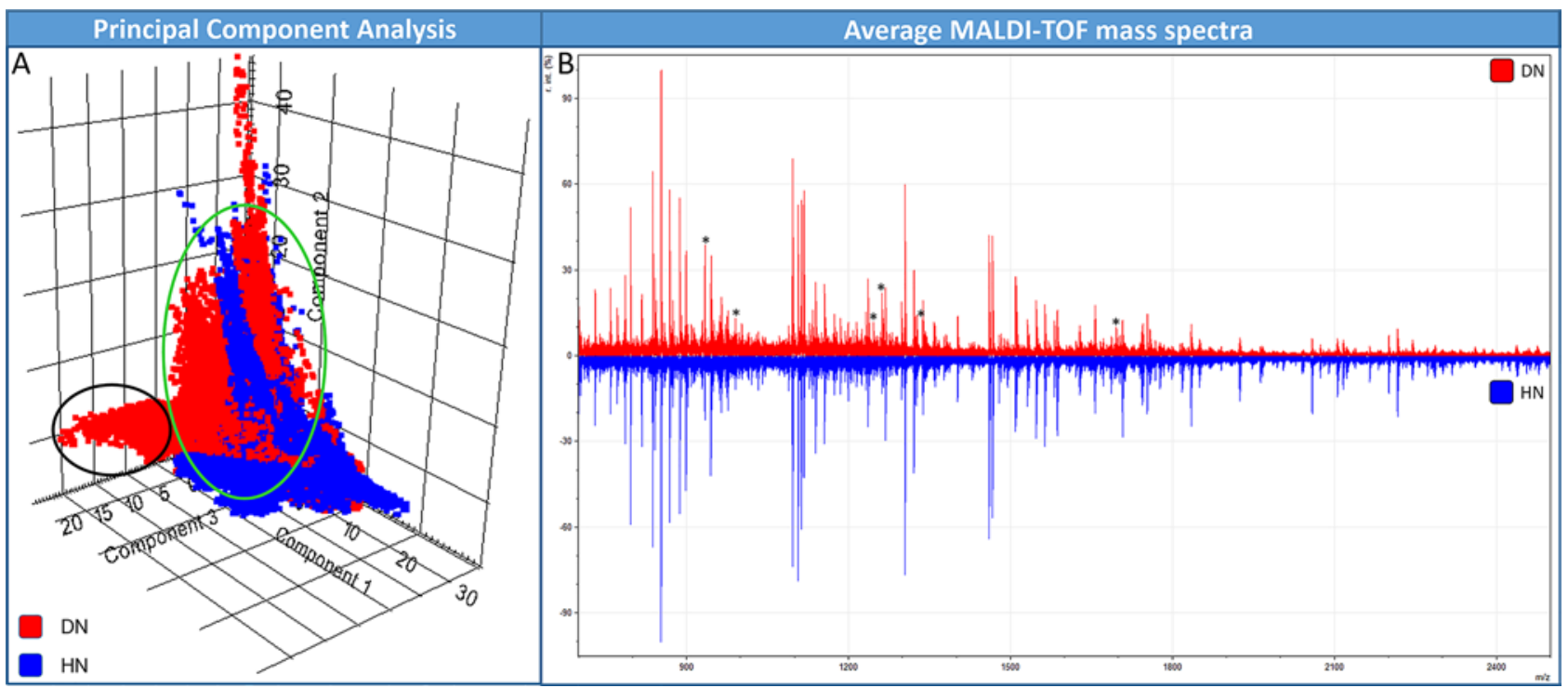

Fig. 2. A Three-dimensional PCA score chart containing spectra obtained from cases of DN and HN. All PCA score charts show the variation present within the first 3 components. B Average tryptic peptide profiles representative of DN and $\mathrm{HN}$ in the mass range of $m / z$ 700-2,500. Relative intensity (r. int.) is expressed as a percentage. Signals highlighted by ROC analysis are denoted by asterisks. 


\section{Kidney \\ Blood Pressure \\ Research}

Table 2. The six $m / z$ signals (AUC $\geq 0.80$ ) detected by ROC analysis when comparing the DN and HN groups

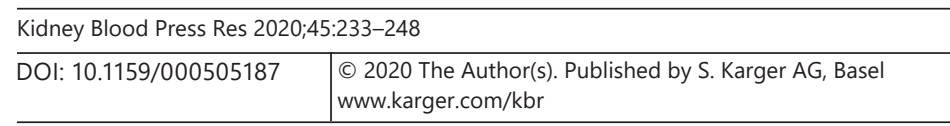

\begin{tabular}{ll}
\hline$m / z$ in MALDI-TOF-MSI & AUC \\
\hline 933.53 & 0.87 \\
$1,001.56$ & 0.85 \\
$1,245.72$ & 0.82 \\
$1,261.75$ & 0.83 \\
$1,357.76$ & 0.82 \\
$1,694.95$ & 0.80 \\
\hline
\end{tabular}

ROC analysis was then performed, comparing the entire DN dataset with the entire HN dataset. A total of six $m / z$ signals, from among the 281 detected $m / z$ features, were observed with an AUC $\geq 0.8$ and a $p \leq 0.05$. These are reported in Table 2 and denoted by asterisks in the average tryptic peptide profiles presented in Figure 2B.

\section{Proteomic Signatures Associated with the Progression of DN}

In order to evaluate the possibility to detect proteins associated with the more rapid advancement of DN, each disease group were separated into mild, moderate, and severe classes following pathological evaluation.

Initially, PCA was performed comparing only those cases that were histologically evaluated as mild from within the DN and HN groups. As presented in Figure 3A, there were clusters of spectra from the mild DN group that shared the same distribution as the spectra from the mild HN group and are indicated by the black circle. Conversely, there were further clusters of spectra from the mild DN group with a distinctly different distribution (red circle), suggestive of additional proteomic alterations that were not observed within the mild HN group. Furthermore, when those cases of HN that were histologically evaluated as moderate were added to the PCA analysis, the spectra from the mild DN group (yellow dots) were clustered closely with the moderate HN group (fuchsia dots), indicating a similar protein profile (black circle in Fig. 3B).

A similar trend was also observed when the moderate and advanced stages of the disease were compared using PCA. As represented in Figure 3C, the spectra obtained from moderate DN were shown to be more similar to those obtained from severe $\mathrm{HN}$, with a region of overlap observed in the first 2 components of the PCA score chart (black circle). Conversely, the spectra deriving from the moderate class of $\mathrm{HN}$, while having a distribution more similar to severe HN than to moderate DN, were distributed in a slightly different region of the PCA score chart. Finally, when the severe cases of both diseases were evaluated (Fig. 3D), the corresponding spectra were well separated, indicating significant differences between their respective tissue proteomes.

The signal intensity of those 6 discriminatory $m / z$ features previously detected (Table 2 ) was also evaluated at each of the disease stages (Fig. 4). In particular, the average signal intensity of $\mathrm{m} / \mathrm{z} 933.53$ and 1,261.75 displayed a similar trend that was represented by a large increase between the mild/moderate stages and the severe stage of DN. This was also further supported by an increased AUC value when comparing the corresponding mild and severe cases of the 2 diseases $(\mathrm{m} / z$ 933.5 - AUC of 0.84 and 0.97 , respectively; $m / z 1,261.75$ - AUC of 0.80 and 0.93 , respectively).

\section{Putative Protein Identification Using MALDI-FTICR MS and nLC-ESI-MS/MS}

A total of 633 and 630 proteins were identified in the pooled DN and HN samples, respectively (data not provided). The obtained accurate masses of the discriminatory $\mathrm{m} / \mathrm{z}$ signals were then correlated with the obtained protein identification list and this led to putative identification of the following 4 proteins: PGRMC1 (progesterone receptor membrane component 
Kidney

Blood Pressure

Research

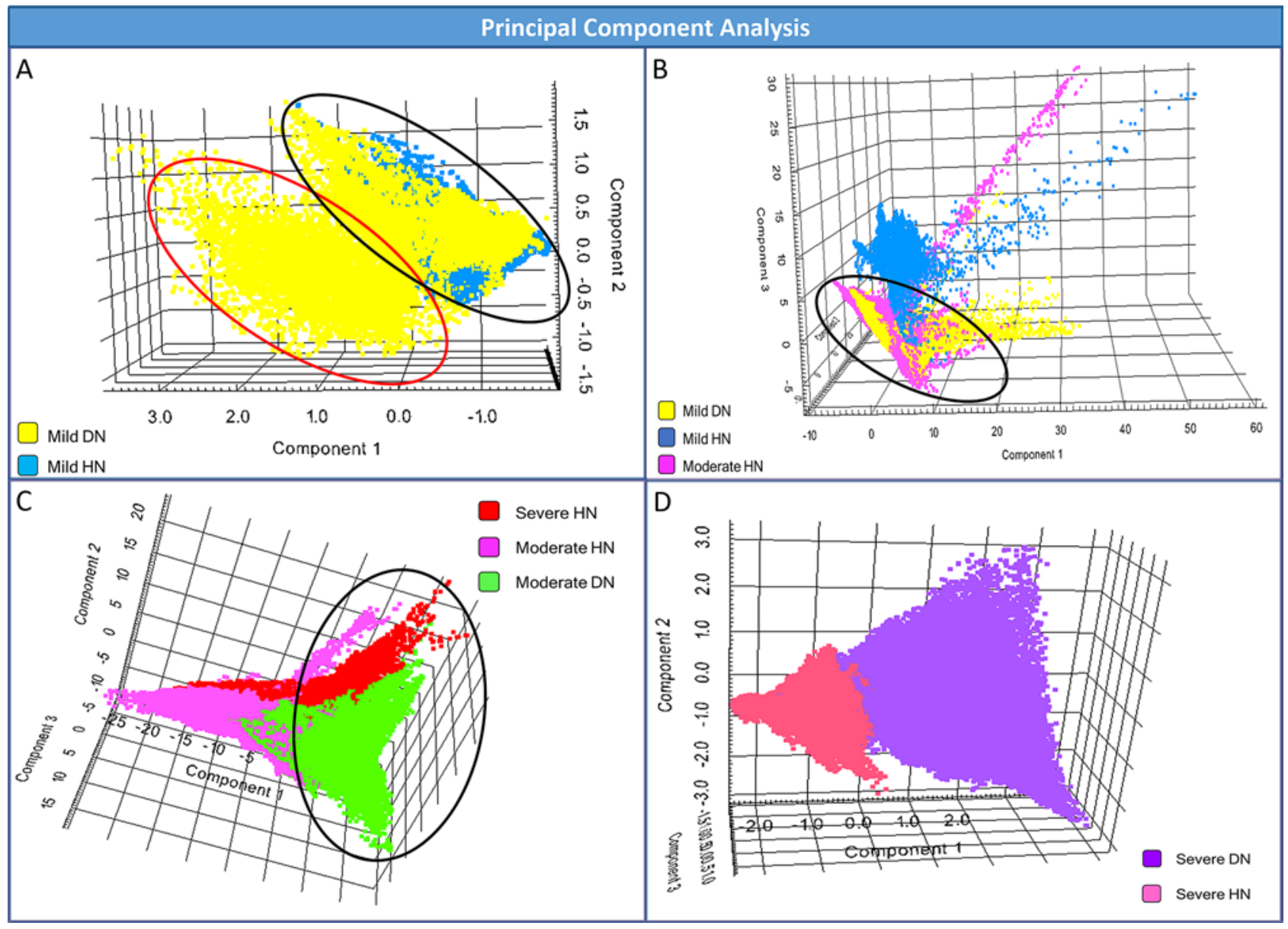

Fig. 3. A-D Three-dimensional PCA score charts containing spectra obtained from the different histological stages of DN and HN. All PCA score charts show the variation present within the first 3 components.

1; peptide sequence: FDGVQDPR), ANXA5 (annexin A5; peptide sequence: VLTEIIASR), CO3 (complement component C3; peptide sequence: QELSEAEQATR), and LDHB (lactate dehydrogenase B; peptide sequence: LIAPVAEEEATVPNNK). This is summarized in Table 3.

\section{Coregistration of MALDI-TOF-MS Images with Histology}

The tissue localization of those putatively identified $m / z$ signals was evaluated. Coregistration of the proteomic MALDI-TOF-MSI information with the PAS-stained histological image highlighted that, within DN renal tissue, the highest signal intensities were observed in tubulointerstitial regions with extensive sclerosis or with a significant accumulation of connective tissue or fibrosis (Fig. 5). While these signals were also present in HN tissue, within tubulointerstitial regions (data not shown), they were of a much lower signal intensity with respect to DN and supportive of the data provided in Figure 4 and Table 1.

\section{Immunohistochemistry Validation of PGRMC1 and CO3}

Quantitative IHC staining for PGRMC1 and CO3 was performed in all the cases analyzed by MALDI-TOF-MSI $(n=18)$. Regarding PGRMC1, the MALDI-TOF-MSI findings were supported and the same localization within the tubulointerstitium was observed. Specifically, there was increased cytoplasmic activity in the tubular cells of the DN cases with respect to $\mathrm{HN}$ cases, 


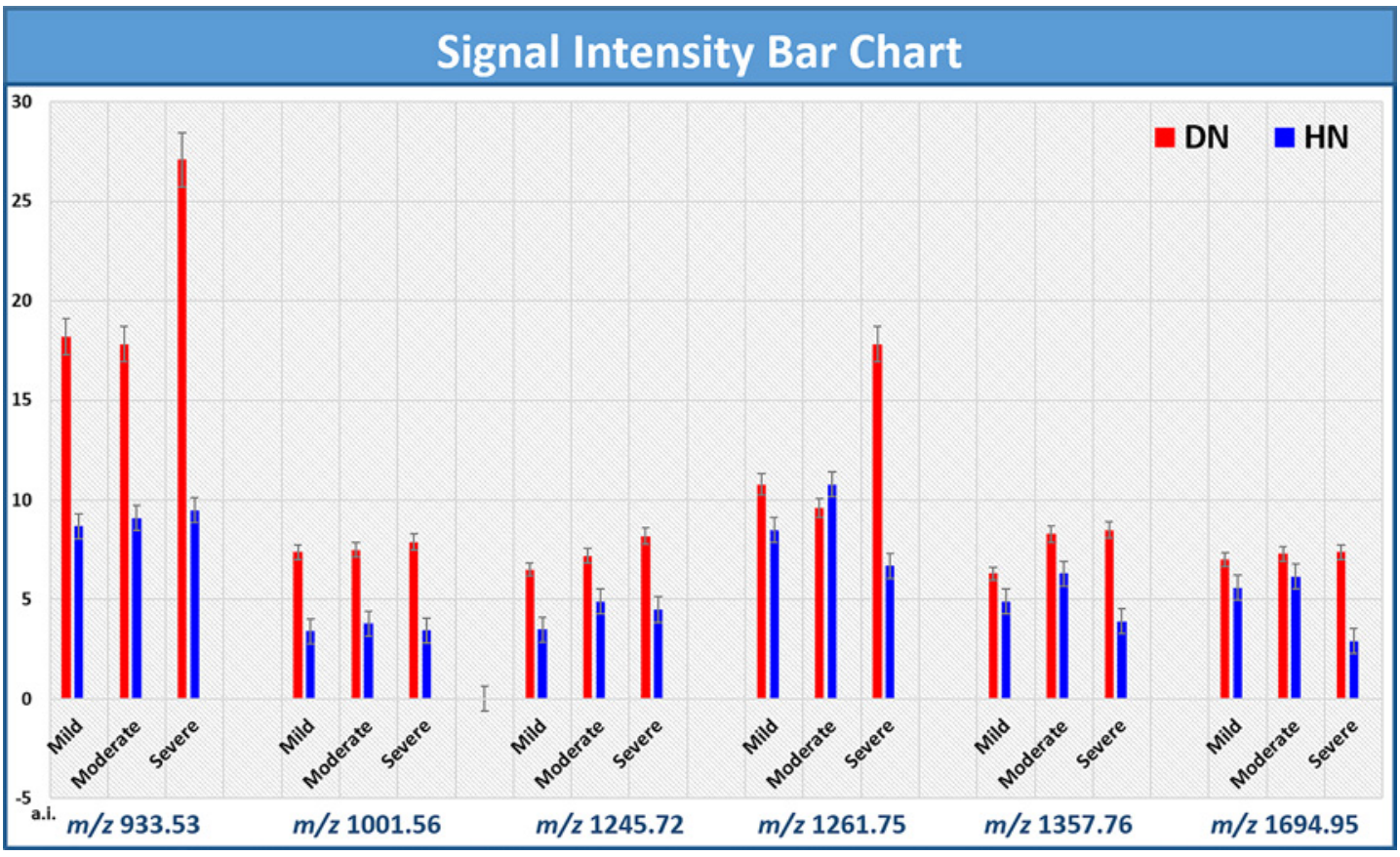

Fig. 4. Intensity bar chart of the $6 \mathrm{~m} / z$ signals that could discriminate between DN and HN at the different histological stages, respectively. Absolute intensity (a.i.) is expressed in arbitrary units. SE bars are included.

Table 3. Putative identification for 4 of the 6 signals detected by ROC analysis following integration of the accurate mass values obtained by MALDI-FTICR MS and nLC-ESI-MS/MS

\begin{tabular}{lrll}
\hline$m / z$ MALDI-TOF-MSI & $m / z$ MALDI-FTICR MS & Putative identity & Error, ppm \\
\hline 933.53 & 933.43960 & PGRMC1_HUMAN & 3.75 \\
$1,001.56$ & $1,001.58776$ & ANXA5_HUMAN & 4.23 \\
$1,245.72$ & $1,245.62079$ & - & - \\
$1,261.75$ & $1,261.6060$ & CO3_HUMAN & 9.51 \\
$1,357.76$ & - & - & 4.78 \\
$1,694.95$ & $1,694.8830$ & LDHB_HUMAN & 4 \\
\hline
\end{tabular}

in particular at the moderate and severe stages, with an increased number of moderate and strong positive pixels being observed (Fig. 6). This difference was not statistically significant and could be attributable to the low patient numbers.

Regarding C03, tissue expression was barely detectable by immunofluorescence probably due to the limitations and sensitivity of antibody-based probes and detection. However, the localization within the tissue compartments was again comparable to that observed in MALDITOF-MSI (data not shown).

\section{Discussion}

The worldwide prevalence of CKD is estimated to be $7.2 \%$ in people aged 30 years or older and the most common causes of CKD in developed countries are diabetes mellitus and hypertension, clinical conditions which often coexist. The inability to differentiate between 
Kidney

Blood Pressure Research

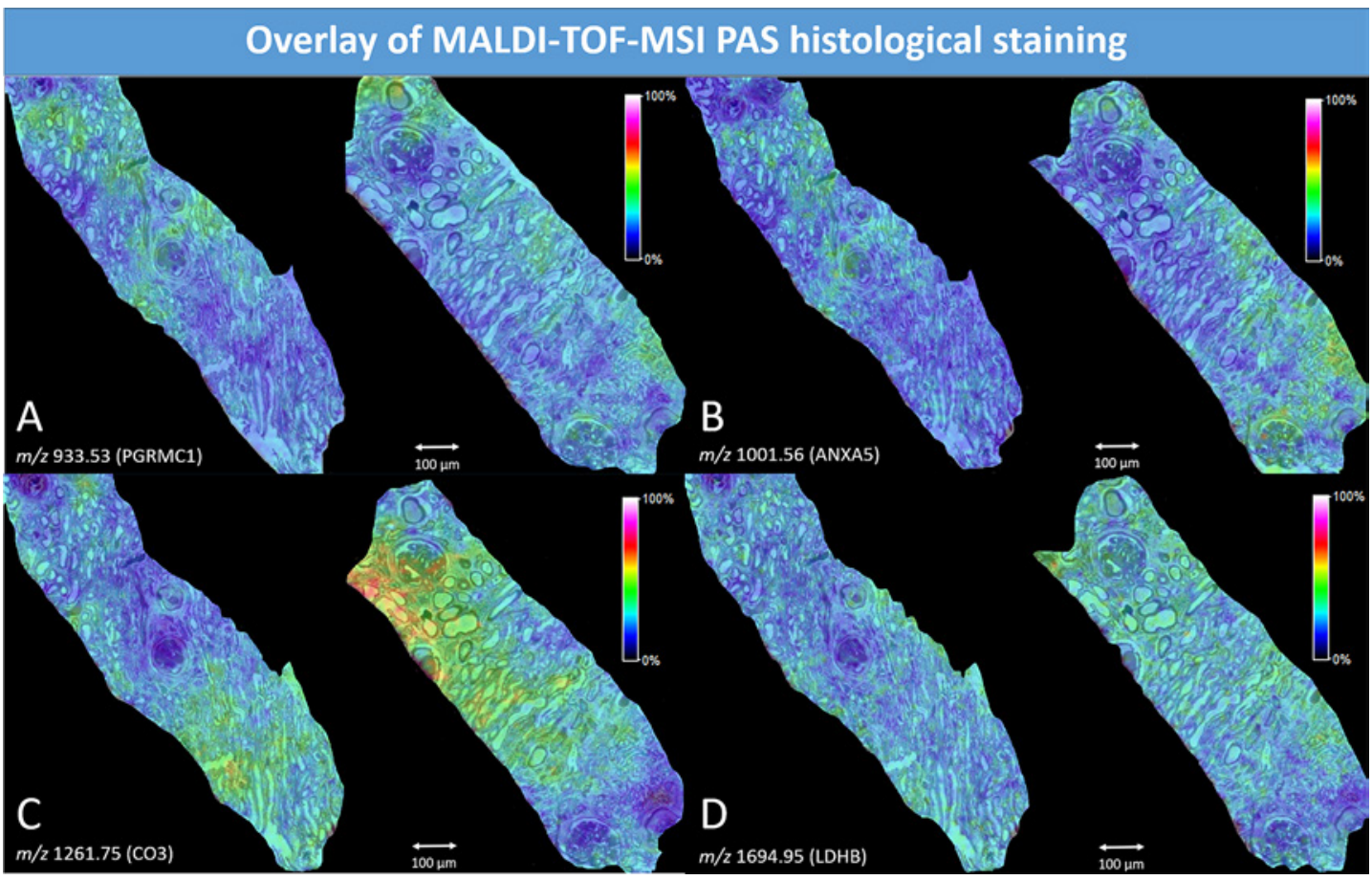

Fig. 5. Exemplary MALDI-TOF-MS images, overlaid with the PAS-stained counterpart, highlighting the spatial distribution of $m / z 933.53$ (PGRMC1) (A); $m / z$ 1,001.56 (ANXA5) (B); $m / z$ 1,261.75 (CO3) (C); and $m / z$ 1,694.95 (LDHB) (D) in DN renal tissue. The highest signal intensities were observed in regions with extensive sclerosis or with a significant accumulation of connective tissue or fibrosis. The intensity color scale is provided.

DN and $\mathrm{HN}$ in patients with both hypertension and diabetes stems from the lack of safe, costeffective, sensitive, and specific clinical biomarkers. Thus, only clinical parameters are used to make treatment and patient care decisions. Therefore, a biomarker that can correctly differentiate between these 2 causes of CKD would lead to targeted therapy and focused monitoring for clinicians. In fact, such a biomarker is highly relevant in the current climate given the success of SGLT2 inhibitors, such as canagliflozin, in significantly slowing down the progression of kidney disease in patients with diabetes [4, 17]. However, a large proportion of participants in the CREDENCE study had hypertension in addition to diabetes, and thus the exact nature of their kidney disease was not confirmed. Identifying patients with DN using a biomarker would allow future studies and clinicians to better select patients in whom SGLT2 inhibitors may be most effective.

After defining the inclusion criteria in base of clinical parameters, high-spatial-resolution MALDI-TOF-MSI was applied in the present series that contained groups of DN and HN. Initially, unsupervised PCA indicated the presence of proteomic differences between the subsets of cells present in the 2 groups (Fig. 2A). These differences were further underlined by performing supervised ROC analysis, highlighting those signals with discriminatory capability (Table 2; Fig. 2B). Further supporting this, these discriminatory signals were also among the key factors considered in the loadings plot of the PCA. When the 2 groups were separated on the basis of their severity following histological evaluation, even greater differences could be observed between the tissue proteome of the 2 diseases (Fig. 3). In particular, the proteomic profiles of mild and moderate DN were more similar to those from the more advanced histological classes of HN (Fig. 3B, C). Together, this data suggests that early stages of DN contain 


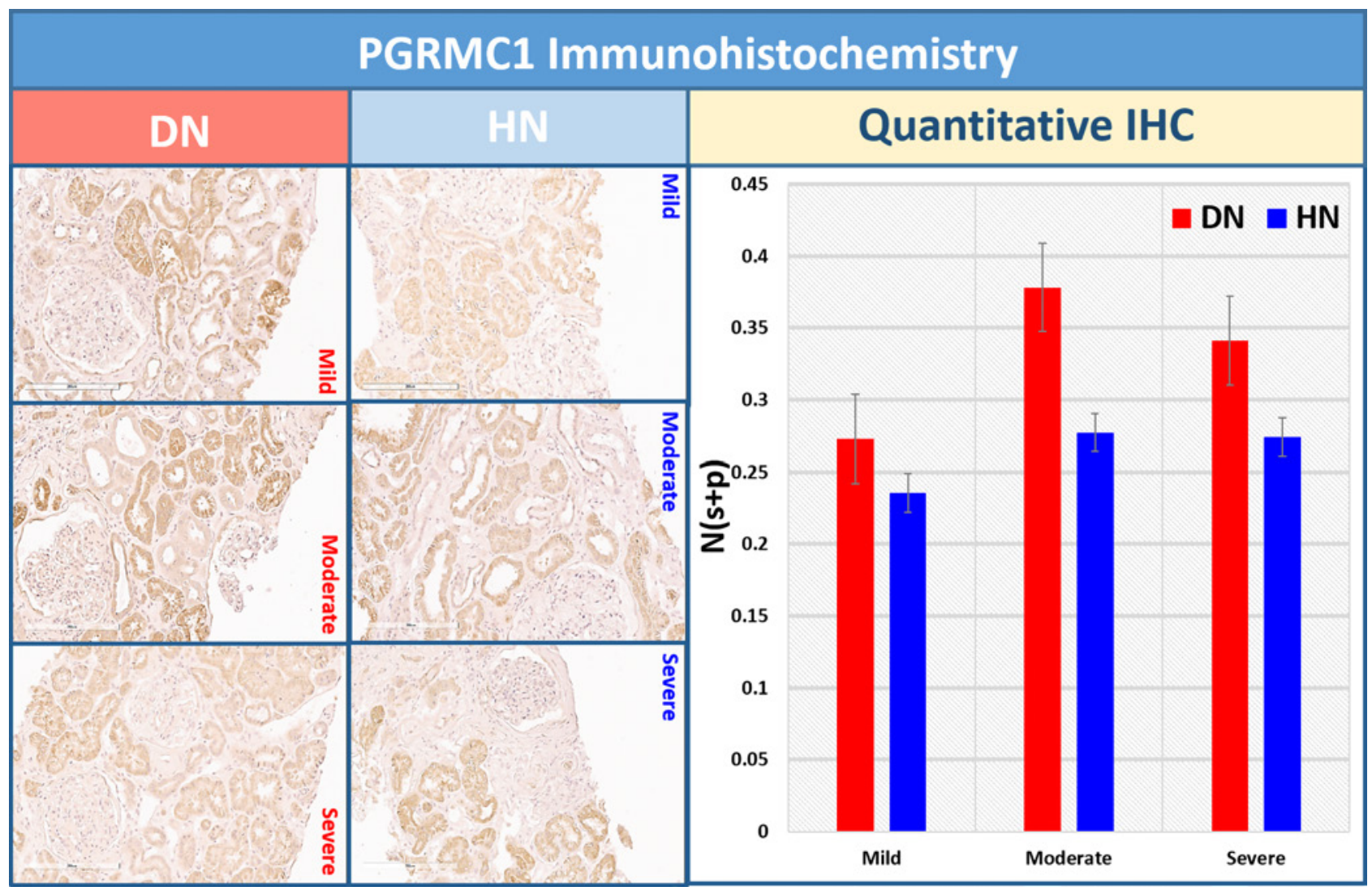

Fig. 6. PGRMC1 immunohistochemistry in DN and HN. Increased cytoplasmic activity could be observed in the tubular cells of the DN cases. Intensity bar chart depicting the output of the quantitative IHC with an increased number of moderate and strong positive pixels $(\mathrm{N}[\mathrm{s}+\mathrm{p}])$ being observed in the different histological classes of DN and HN, in particular at the moderate and severe stages. SE bars are included.

pathophysiologic derangements that are seen in more severe stages of HN. This observation is consistent with increased clinical disease severity of DN compared to HN and the propensity for more rapid progression to kidney failure. Furthermore, given that these alterations may also be detected at an earlier stage with respect to routine renal histology, this may enable earlier and more correct clinical patient management [18].

Following integration of the MALDI-TOF-MSI data with that of MALDI-FTICR MS and nLCESI-MS/MS, the following 4 signals that could discriminate between DN and HN were putatively identified: PGRMC1 ( $\mathrm{m} / z$ 933.53), ANXA5 ( $\mathrm{m} / z$ 1,001.56), CO3 ( $\mathrm{m} / z$ 1,261.75), and LDHB $(m / z$ 1,694.95). When the tissue localization of these signals was evaluated, all were found to be of a higher intensity in areas of tissue with large amounts of glomerulosclerosis or an accumulation of connective tissue and fibrosis within the tubulointerstitium (Fig. 5), histological features which commonly correspond with the more severe stage of disease development. This may be particularly relevant in DN given that there is already prior evidence to suggest that renal fibrosis, as a result of the accumulation of connective tissue, is induced by the production and deposition of advanced glycation end products [19] and can be associated with poorer renal outcomes [20]. When the signal intensity of these proteins were evaluated within the different histological groups of DN, PGRMC1 and CO3 showed a marked increase in signal intensity in the severe class with respect to the mild and moderate classes (Fig. 4), where the presence of fibrotic and sclerotic tissue is more prevalent [21], 


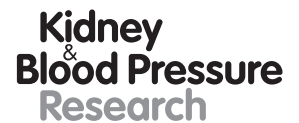

Kidney
Blood Pressure

Research \begin{tabular}{l|l}
\hline Kidney Blood Press Res 2020;45:233-248 \\
\hline DOI: 10.1159/000505187 & $\begin{array}{l}\text { @ 2020 The Author(s). Published by S. Karger AG, Basel } \\
\text { www.karger.com/kbr }\end{array}$ \\
\hline
\end{tabular}

Smith et al:: MALDI-MSI to Distinguish DN from HN

providing a tentative suggestion that the increased detection of these proteins may be associated with a poorer renal outcome.

The finding of increased $\mathrm{CO} 3$ is not surprising since complement activation is increasingly recognized as an effector mechanism during tissue injury in various forms of CKD including DN [22]. While DN has historically been considered a non-immune-mediated renal disease, there is emerging data to suggest that the activation of the complement cascade may contribute to the development of DN and it may also explain the rapid progression of DN with respect to $\mathrm{HN}[23,24]$. In particular, overexpression of C3 has been detected in renal tissue of DN with respect to controls in multiple animal models, with an even larger overexpression in those animals with induced ischemia [25]. However, $\mathrm{CO} 3$ was not clearly detected in this DN patient cohort using standard immunofluorescence, primarily due to the limited sensitivity of antibody based probes and detection. Nevertheless, this data provides further insight into the mechanisms that may underlie DN versus HN progression in humans. In light of this, the role of C3 in human DN could be further investigated, especially in tubulointerstial compartments, in order to verify its utility in the distinction between diabetic and hypertensive causes of CKD.

Upon quantitative IHC validation, PGRMC1 was observed to be localized to the tubulointerstitium, as observed by MALDI-TOF-MSI. Furthermore, there was increased cytoplasmic activity in the tubular cells of the DN cases. While the same marked increase in tissue positivity was not observed in the severe group, this is not to be unexpected given the differing levels of sensitivity and specificity between MALDI-MS based techniques and IHC [26, 27]. Irrespectively of this, the detection of increased levels of PGRMC1 in DN, using both techniques, still holds promise in this clinical context. PGRMC1 (progesterone receptor membrane component 1) is implicated in diverse cellular processes, including proliferation and resistance to apoptosis [28]. Furthermore, it is commonly overexpressed in many cancers including ovarian, breast, and kidney [29-31] and its expression in human tumors may be triggered by hypoxia [32].

Focusing on the expression of PGRMC1 in the kidney, our results confirm previous findings localizing PGRMC1 to the cytoplasm of tubular epithelial cells in the kidney [31]. Zhang et al. [31] showed that renal cell carcinomas highly expressed PGRMC1. Furthermore, increased levels of PGRMC1 were found in the sera of renal cell carcinoma patients and they were associated with a worse survival. While the authors did not speculate on the driving force behind its expression, PGRMC1 is likely induced by the hypoxic tumor milieu in renal cell carcinoma. Interestingly, the tubulointerstitial damage seen in DN is thought to be, in part, due to tissue hypoxia. Chronic ischemia is the primary cause of tissue hypoxia in DN and it is thought to arise in 2 ways [33]. First, intrarenal vasoconstriction due to RAS activation or decreased NO activity can limit blood flow and decrease oxygen delivery. Second, blood flow may be limited structurally by the presence of interstitial fibrosis surrounding the peritubular capillaries. Thus, increased PGRMC1 expression may represent worsening hypoxia in DN and its increased intensity in regions with interstitial fibrosis is supportive of this hypothesis.

If the tissue findings related to these 2 proteins (CO3 and PGRMC1) is confirmed in a larger cohort of patients, it may then be relevant to follow their expression in the urine of patients with DN and HN. Complement component C3 has already been detected in the urine of patients with DN and, in fact, its abundance was negatively correlated with eGFR and associated with poorer renal outcomes [34]. Furthermore, it has been shown that urinary excretion of PGRMC1 may be indicative of its direct kidney origin [35] and, in particular, altered levels of PGRMC1 were detected in the urine of patients with type 1 and type 2 diabetes with respect to controls [36]. Therefore, given the potential to detect these proteins in urine, they may represent possible targets to distinguish diabetic and hypertensive forms of kidney disease using a noninvasive approach. 


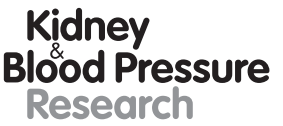

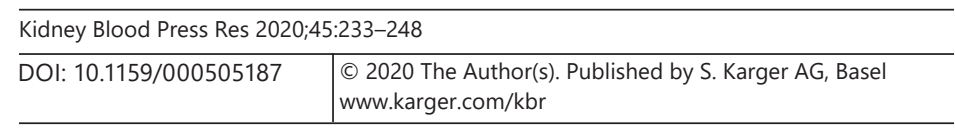

Smith et al.: MALDI-MSI to Distinguish DN from HN

On the contrary, while a number of studies have also demonstrated the increased excretion of collagen fragments in patients with DN [36,37], we did not detect differences in collagen expression between DN and HN. However, collagen $\alpha-1$ chains, for example, are expressed as general markers of fibrosis and may not represent a useful marker to distinguish between DN and HN given that renal fibrosis is a common pathway and hallmark of all CKD progression [38]. Furthermore, if the spatially resolved analysis of extracellular matrix proteins are to be correctly investigated using this approach, the use of matrix metalloproteinases, such as collagenase type III or elastase, which specifically target collagens would represent the more efficient strategy [39]. Unfortunately, this was outside of the scope of this study.

While our case series was defined by using primarily pathological criteria, and can thus reduce possible confounding factors in the analysis, this can often limit the size of the sample cohort and the low number of patients in each group represents the largest shortcoming of this study. Furthermore, clinical information related to proteinuria and albuminuria is not available for all patients and represents another pitfall of this study. Therefore, there is the need for a larger sample cohort to confirm the hypothesis of this study, especially with regard to evaluating the significance of these findings with respect to disease progression. However, this limitation notwithstanding, this preliminary study highlights the feasibility of integrating high-spatial-resolution MALDI-MSI with high-mass accuracy MS in order to search for novel kidney-derived protein markers of DN. The cluster of putative proteins presented here represents a valuable starting point for a future study employing a larger cohort of patients and, if verified, it would allow for the development of clinical biomarkers in blood or urine that would assist the clinician in providing more precise therapy for patients with DN and HN.

\section{Acknowledgement}

Our special thanks go to Kevin Chapman and Michelle Nelson for their technical and administrative support of this study.

\section{Statement of Ethics}

The authors confirm that this research complies with the relevant ethics guidelines. Tissue samples in the BMCKD database represent the secondary use of specimens collected for clinical care under a protocol approved by the Conjoint Health Research Ethics Board at the University of Calgary. Samples are coded and do not contain any patient-identifying data, and thus patient consent was not required.

\section{Disclosure Statement}

The authors state that they have no conflict of interests.

\section{Funding Sources}

The research leading to these results received funding from MIUR: FIRB 2007 (RBRN07BMCT_11), FAR 2014-2016, and in part the Fondazione Gigi \& Pupa Ferrari Onlus. 


\section{Kidney \\ Blood Pressure \\ Research}

\begin{tabular}{l|l}
\hline Kidney Blood Press Res 2020;45:233-248 \\
\hline DOI: 10.1159/000505187 & $\begin{array}{l}\text { @ 2020 The Author(s). Published by S. Karger AG, Basel } \\
\text { www.karger.com/kbr }\end{array}$ \\
\hline
\end{tabular}

Smith et al.: MALDI-MSI to Distinguish DN from HN

\section{Author Contributions}

All of the authors contributed to the production of this manuscript in accordance with the ICMJE Criteria for Authorship.

\section{References}

1 Jha V, Garcia-Garcia G, Iseki K, Li Z, Naicker S, Plattner B, et al. Chronic kidney disease: global dimension and perspectives [Internet]. Lancet. 2013 Jul;382(9888):260-72.

2 Woroniecka KI, Park AS, Mohtat D, Thomas DB, Pullman JM, Susztak K. Transcriptome analysis of human diabetic kidney disease. Diabetes. 2011 Sep;60(9):2354-69.

3 Furuichi K, Shimizu M, Okada H, Narita I, Wada T. Clinico-pathological features of kidney disease in diabetic cases. Clin Exp Nephrol. 2018 Oct;22(5):1046-51.

4 Perkovic V, Jardine MJ, Neal B, Bompoint S, Heerspink HJ, Charytan DM, et al. Canagliflozin and Renal Outcomes in Type 2 Diabetes and Nephropathy. N Engl J Med. 2019 Jun 13;380(24):2295-306.

5 Adler AI, Stevens RJ, Manley SE, Bilous RW, Cull CA, Holman RR; UKPDS GROUP. Development and progression of nephropathy in type 2 diabetes: the United Kingdom Prospective Diabetes Study (UKPDS 64). Kidney Int. 2003 Jan;63(1):225-32.

6 Vanholder R, Annemans L, Brown E, Gansevoort R, Gout-Zwart JJ, Lameire N, et al.; European Kidney Health Alliance. Reducing the costs of chronic kidney disease while delivering quality health care: a call to action [Internet]. Nat Rev Nephrol. 2017 Jul;13(7):393-409.

7 Korbet SM, Volpini KC, Whittier WL. Percutaneous renal biopsy of native kidneys: a single-center experience of 1,055 biopsies. Am J Nephrol. 2014;39(2):153-62.

8 Rossing K, Mischak H, Dakna M, Zürbig P, Novak J, Julian BA, et al.; PREDICTIONS Network. Urinary proteomics in diabetes and CKD. J Am Soc Nephrol. 2008 Jul;19(7):1283-90.

9 Thongboonkerd V. Practical points in urinary proteomics. J Proteome Res. 2007 Oct;6(10):3881-90.

10 Smith A, Piga I, Galli M, Stella M, Denti V, Del Puppo M, et al. Matrix-assisted laser desorption/ionisation mass spectrometry imaging in the study of gastric cancer: A mini review. Int J Mol Sci. 2017 Dec;18(12):E2588.

11 L'Imperio V, Smith A, Chinello C, Pagni F, Magni F. Proteomics and glomerulonephritis: A complementary approach in renal pathology for the identification of chronic kidney disease related markers. Proteomics Clin Appl. 2016 Apr;10(4):371-83.

12 Smith A, L'Imperio V, De Sio G, Ferrario F, Scalia C, Dell'Antonio G, et al. $\alpha$-1-Antitrypsin detected by MALDI imaging in the study of glomerulonephritis: its relevance in chronic kidney disease progression. Proteomics. 2016 Jun;16(11-12):1759-66.

13 Smith A, L'Imperio V, Ajello E, Ferrario F, Mosele N, Stella M, et al. The putative role of MALDI-MSI in the study of Membranous Nephropathy. Biochim Biophys Acta Proteins Proteomics. 2017 Jul;1865(7):865-74.

14 Smith A, L'Imperio V, Denti V, Mazza M, Ivanova M, Stella M, et al. High Spatial Resolution MALDI-MS Imaging in the Study of Membranous Nephropathy. Proteomics Clin Appl. 2019 Jan;13(1):e1800016.

15 Muruve DA, Mann MC, Chapman K, Wong JF, Ravani P, Page SA, et al. The biobank for the molecular classification of kidney disease: research translation and precision medicine in nephrology. BMC Nephrol. 2017 Jul; 18(1):252.

16 De Sio G, Smith AJ, Galli M, Garancini M, Chinello C, Bono F, et al. A MALDI-Mass Spectrometry Imaging method applicable to different formalin-fixed paraffin-embedded human tissues. Mol Biosyst. 2015 Jun;11(6):150714.

17 Guthrie R. Canagliflozin and cardiovascular and renal events in type 2 diabetes. Postgrad Med. 2018 Mar; 130(2):149-53.

18 L'Imperio V, Smith A, Ajello E, Piga I, Stella M, Denti V, et al. MALDI-MSI Pilot Study Highlights Glomerular Deposits of Macrophage Migration Inhibitory Factor (MIF) as a Possible Indicator of Response to Therapy in Membranous Nephropathy. Proteomics Clin Appl. 2019 May;13(3):e1800019.

19 Sugimoto K, Yasujima M, Yagihashi S. Role of advanced glycation end products in diabetic neuropathy. Curr Pharm Des 2008;14(10):953-61.

20 Yamagishi S, Matsui T. Advanced glycation end products, oxidative stress and diabetic nephropathy. Oxid Med Cell Longev. 2010 Mar-Apr;3(2):101-8.

21 Tervaert TW, Mooyaart AL, Amann K, Cohen AH, Cook HT, Drachenberg CB, et al.; Renal Pathology Society. Pathologic classification of diabetic nephropathy. J Am Soc Nephrol. 2010 Apr;21(4):556-63.

22 Sun ZJ, Li XQ, Chang DY, Wang SX, Liu G, Chen M, et al. Complement deposition on renal histopathology of patients with diabetic nephropathy. Diabetes Metab. 2019 Sep;45(4):363-68.

23 Flyvbjerg A. The role of the complement system in diabetic nephropathy. Nat Rev Nephrol. 2017 May;13(5): 311-8.

24 Zhang J, Wang Y, Zhang R, Li H, Han Q, Guo R, et al. Implication of decreased serum complement 3 in patients with diabetic nephropathy. Acta Diabetol. 2018 Jan;55(1):31-9.

25 Kelly KJ, Liu Y, Zhang J, Dominguez JH. Renal C3 complement component: feed forward to diabetic kidney disease. Am J Nephrol. 2015;41(1):48-56. 
26 KRIEGSMANN J. KRIEGSMANN M, CASADONTE R. MALDI TOF imaging mass spectrometry in clinical pathology: A valuable tool for cancer diagnostics (review). Int J Oncol. 2015 Mar;46(3):893-906.

27 Winter M, Tholey A, Kristen A, Röcken C. MALDI Mass Spectrometry Imaging: A Novel Tool for the Identification and Classification of Amyloidosis. Proteomics. 2017 Nov;17(22):1-9.

28 Ahmed IS, Rohe HJ, Twist KE, Craven RJ. Pgrmc1 (progesterone receptor membrane component 1) associates with epidermal growth factor receptor and regulates erlotinib sensitivity. J Biol Chem. 2010 Aug;285(32): 24775-82.

29 Peluso JJ, Liu X, Saunders MM, Claffey KP, Phoenix K. Regulation of ovarian cancer cell viability and sensitivity to cisplatin by progesterone receptor membrane component-1. J Clin Endocrinol Metab. 2008 May; 93(5): 1592-9.

30 Crudden G, Loesel R, Craven RJ. Overexpression of the cytochrome p450 activator hpr6 (heme-1 domain protein/human progesterone receptor) in tumors. Tumour Biol. 2005 May-Jun;26(3):142-6.

31 Zhang D, Xia X, Wang X, Zhang P, Lu W, Yu Y, et al. PGRMC1 is a novel potential tumor biomarker of human renal cell carcinoma based on quantitative proteomic and integrative biological assessments. PLoS One. 2017 Jan;12(1):e0170453.

32 Dressman HK, Hans C, Bild A, Olson JA, Eric Rosen P, Marcom K, et al. Gene expression profiles of multiple breast cancer phenotypes and response to neoadjuvant chemotherapy. Clin Cancer Res. 2006;12(3 I):819-26.

33 KanwarYS, Sun L, Xie P, Liu FY, Chen S. A glimpse of various pathogenetic mechanisms of diabetic nephropathy. Annu Rev Pathol. 2011;6(7):395-423.

34 Vaisar T, Durbin-johnson B, Whitlock K, Babenko I, Mehrotra R, Rocke DM. Urine Complement Proteins and the Risk of Kidney Disease Progression and Mortality in Type 2 Diabetes. Diabetes Care. 2018;41(11):236169.

35 Magalh P, Pontillo C, Pejchinovski M, Siwy J, Krochmal M, Makridakis M, et al. Comparison of Urine and Plasma Peptidome Indicates Selectivity in Renal Peptide Handling. Proteomies Clin Appl. 2018;12(5):e1700163.

36 Maahs DM, Siwy J, Argilés A, Cerna M, Delles C, Dominiczak AF, et al. Urinary collagen fragments are significantly altered in diabetes: a link to pathophysiology. PLoS One. 2010 Sep;5(9):e13051.

37 Alkhalaf A, Zürbig P, Bakker SJ, Bilo HJ, Cerna M, Fischer C, et al.; PREDICTIONS Group. Multicentric validation of proteomic biomarkers in urine specific for diabetic nephropathy. PLoS One. 2010 Oct;5(10):e13421.

38 Liu Y. Cellular and molecular mechanisms of renal fibrosis. Nat Rev Nephrol. 2011 Oct; 7(12):684-96.

39 Angel PM, Comte-Walters S, Ball LE, Talbot K, Mehta A, Brockbank KG, et al. Mapping Extracellular Matrix Proteins in Formalin-Fixed, Paraffin-Embedded Tissues by MALDI Imaging Mass Spectrometry. J Proteome Res. 2018 Jan;17(1):635-46. 